\title{
Memristor-based Synapse Design and Training Scheme for Neuromorphic Computing Architecture
}

\author{
Hui Wang and Hai (Helen) Li \\ Department of Electrical and Computer Engineering \\ Polytechnic Institute of New York University \\ Brooklyn, New York, USA \\ hwang07@students.poly.edu, hli@poly.edu
}

\author{
Robinson E. Pino \\ Air Force Research Laboratory, Information Directorate \\ Advanced Computing Architectures \\ Rome, New York, USA \\ robinson.pino@rl.af.mil
}

\begin{abstract}
Memristors have been rediscovered recently and then gained increasing attentions. Their unique properties, such as high density, nonvolatility, and recording historic behavior of current (or voltage) profile, have inspired the creation of memristor-based neuromorphic computing architecture. Rather than the existing crossbar-based neuron network designs, we focus on memristor-based synapse and the corresponding training circuit to mimic the real biological system. In this paper, first, the basic synapse design is presented. On top of it, we will discuss the training sharing scheme and explore design implication on multi-synapse neuron system. Energy saving method such as self-training is also investigated.
\end{abstract}

Keywords - memristor; synapse; training

\section{INTRODUCTION}

The neuromorphic computing architecture that requires a large volume of memory and being adaptive to environment has demonstrated great potentials in developing high performance parallel computing systems [1]. Currently, most of research activities have been conducted at software or system level built upon the conventional Von Neumann computer architecture [2][3]. Developing the neuromorphic architecture at chip level by mimicking the biological system is another important direction. However, it results in high design complex and cost by using the traditional CMOS devices.

Though the existence of memristor was predicted in 1971 [4], the first physical realization was first reported thirty years later by $\mathrm{HP}$ Lab through a $\mathrm{TiO}_{2}$ thin-film device[5]. Afterwards, more materials with memristive properties have been reported or rediscovered. The unique properties of memristor make it very promising to be used to mimic natural neuron networks [7]. First, the memristor-based memory can achieve an integration density as high as $10 \mathrm{Gbits} / \mathrm{cm}^{2}$ [4][5]. Second, the memristor device has an intrinsic and remarkable feature called "pinched hysteresis loop", that is, the memristance relies on the total electric charge flowing through it [4][6]. Third, memristance remains unchanged when power is turned off.
Many memristor-based circuit designs have been explored, such as crossbar nonvolatile memory [8] and FPGA [9]. Strukov et al. integrated digital memory, programmable Boolean logic circuit and also neuron networks within a 3D hybrid CMOS/memristor structure. Rajendran et al. proposed memristor-based programmable threshold logic array [11] utilized it in synapse-neuron structure [12]. However, the training circuit and training scheme for memristor-based reconfigurable architecture design have not been fully explored yet.

In this paper, we proposed a single memristor-based synapse structure and the corresponding training circuit design. On top of it, we discussed the design optimization and its implementation in multi-synapse systems. With the aid of sharing training circuit and self-training mode, the performance and energy can be significantly improved. In the paper, we demonstrate the effective of the proposed synapse design by using $\mathrm{TiO}_{2}$ memristor, though the design philosophy can be generalized to other memristor materials.

\section{PRELIMINARY}

In 1971, Professor Chua predicted the existence of the fourth fundamental circuit element, named as memristor, that uniquely builds the relationship between the magnetic flux $(\varphi)$ and the electric charge $(q)$ passing through the device as [4]:

$$
d \varphi=M \cdot d q
$$

Considering that magnetic flux and the electric charge are integrals of voltage $(V)$ and current $(I)$ over time,

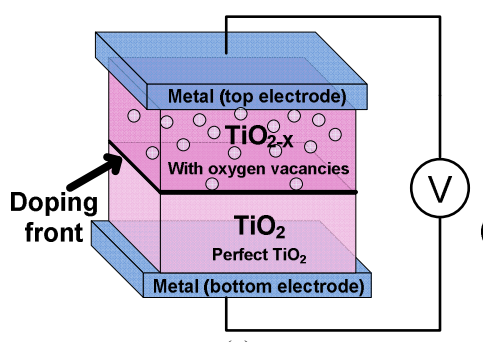

(a)

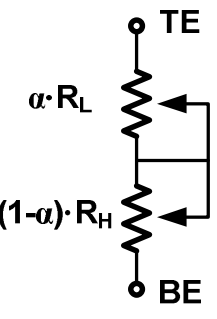

(b)
Figure 1: $\mathrm{TiO}_{2}$ thin-film memristor. (a) Structure. (b) Equivalent circuit. 
respectively. Eq. (1) can be expressed as

$$
\left\{\begin{array}{l}
V=M(\omega, I) \cdot I \\
d \omega / d t=f(\omega, I)
\end{array},\right.
$$

where, $M(\omega, I)$ represents instantaneous memristance which varies over time and $\omega$ is a state variable.

Figure 1(a) illustrates the conceptual view of $\mathrm{Pt} / \mathrm{TiO}_{2} / \mathrm{Pt}$ structure: two metal wires $(\mathrm{Pt})$ construct the top and bottom electrodes, and a thick titanium dioxide film is sandwiched in between. A perfect $\mathrm{TiO}_{2}$ structure has a natural state as an insulator. However, the conductivity of oxygen-deficient titanium dioxide $\left(\mathrm{TiO}_{2-\mathrm{x}}\right)$ is much higher. By moving the doping front under proper electrical excitations, the intermediate memristive state can be achieved. We use $R_{H}$ and $R_{L}$ to denote the total resistance when a $\mathrm{TiO}_{2}$ memristor is fully undoped and doped, respectively. The overall memristance can be equivalent as two serially-connected resistances, as shown in Figure 1(b). That is

$$
M(\alpha)=\alpha \cdot R_{L}+(1-\alpha) \cdot R_{H} .
$$

Here, $\alpha(0 \leq \alpha \leq 1)$ is the relative doping front position, which is the ratio of doping front position over the total thickness of a $\mathrm{TiO}_{2}$ device.

For demonstration purpose, we built our design based on $\mathrm{TiO}_{2}$ thin-film memristor and adopted the device parameters from [5]. The memristance range is set from $R_{L}=1 \mathrm{~K} \Omega$ to $R_{H}=16 \mathrm{~K} \Omega$. Note that the proposed can be applied to many memristor materials with different physical mechanisms.

\section{THE PRINCIPLE OF MEMRISTOR-BASED SYNAPSE}

Rather than using memristor crossbar array in neuromorphic reconfigurable architecture, we propose a memristorbased synapse design to mimic the biological structure. Figure 2(a) depicts the conceptual scheme, which simply consists of a NMOS transistor $(Q)$ and a memristor. When the input $V_{\text {in }}$ is low, $Q$ is turned off and the output $V_{\text {out }}$ is connected to ground through the memristor. On the contrary, when $V_{i n}$ is high and turns on $Q$, memristance $M$ and the equivalent resistance of $Q\left(R_{\mathrm{Q}}\right)$ together determine $V_{\text {out }}$ :

$$
V_{\text {out }}=f\left(V_{\text {in }} \cdot M\right) \text {. }
$$

Here, $V_{\text {out }}$ is weighted by the memristance, which behaves like a synapse. Figure 2(b) shows the simulated $V_{\text {out }}$ when sweeping the memristance from $1 \mathrm{~K} \Omega$ to $16 \mathrm{~K} \Omega$. Here,

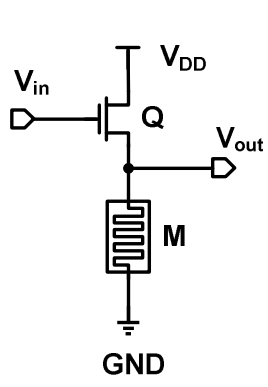

(a)

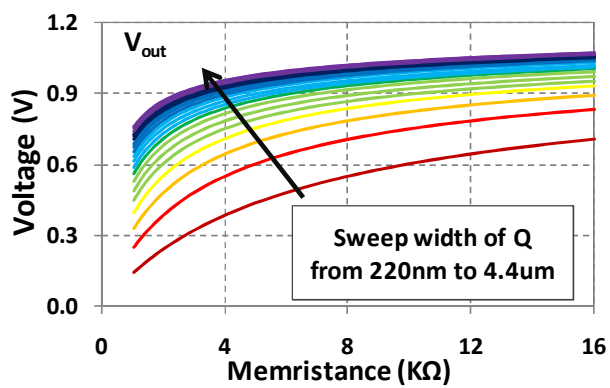

(b)
Figure 2: (a) Proposed synapse design. (b) Synapse output vs. memristance.

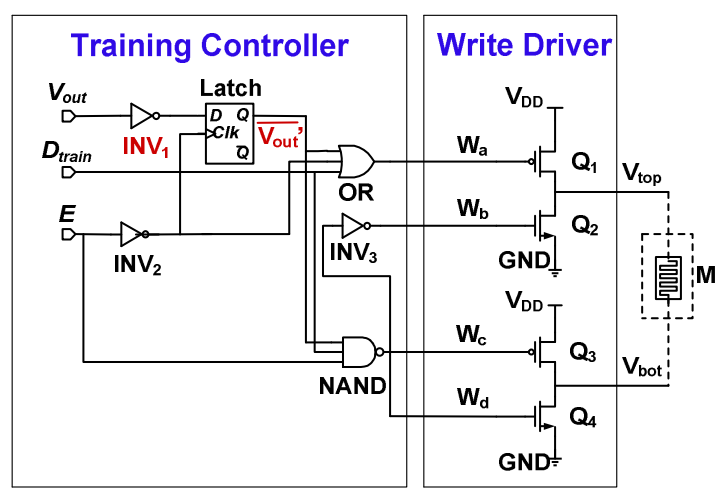

(a)

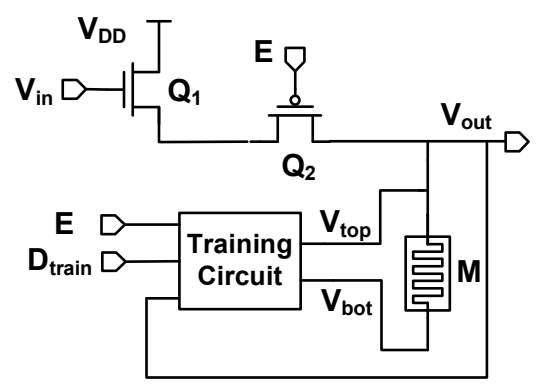

(b)

Figure 3: (a) The training circuit diagram. (b) The proposed synapse togteher with training circuit.

CMOS devices used TSMC $0.18 \mu \mathrm{m}$ technology.

Note that the response of the synapse design is dependent on the equivalent resistance of the transistor $Q\left(R_{\mathrm{Q}}\right)$, or, the size of $Q$. This can also be demonstrated in Figure 2(b) by sweeping the width of $Q$ from $220 \mathrm{~nm}$ to $4.4 \mu \mathrm{m}$ with a step of $220 \mathrm{~nm}$. The simulation shows that a larger $Q$ can result in a wider range of $V_{\text {out }}$ with poorer linearity. However, for a large $Q$, the enhancement of $V_{\text {out }}$ by further increasing its size is marginal.

To improve design stability, a buffer can be added at output of the synapse to increase voltage swing. Furthermore, some circuit optimization techniques, such as asymmetry gate in other blocks, can be used to minimize the overall synapsebased system, as we shall show in Section IV.

\section{SyNAPSE TRAINING CiRCUIT}

\section{A. Synapse Training Circuit Design}

Being self-adaptive to the environment is one of the most important properties of a biological synapse. To accomplish the similar functionality, a training block is needed in the memristor-based synapse that can adjust its memristance.

\section{1) Memristor Training Circuit}

Figure 3(a) shows the diagram of training circuit for one synapse design, based on logic analysis and simplification. It includes two major components: training controller and write driver. By comparing the current synapse output $V_{\text {out }}$ and the expected output $D_{\text {train }}$, training controller generates the control 
TABLE I. TRAINING CIRCUIT OPERATION CONDITIONS.

\begin{tabular}{c|c|c|c|c|c|c}
\hline $\boldsymbol{E}$ & $\boldsymbol{V}_{\text {out }}$ & $\boldsymbol{D}_{\text {train }}$ & $\boldsymbol{V}_{\text {top }}$ & $\boldsymbol{V}_{\text {bot }}$ & $\boldsymbol{V}_{\text {mem }}$ & Status \\
\hline \hline 0 & $\mathrm{X}$ & $\mathrm{X}$ & Floating & 0 & $\mathrm{X}$ & Operating \\
1 & $1 / 0$ & $1 / 0$ & 0 & 0 & $0 \mathrm{~V}$ & No training \\
1 & 1 & 0 & 1 & 0 & $1.8 \mathrm{~V}$ & $\mathrm{R}_{\mathrm{H}}$ to $\mathrm{R}_{\mathrm{L}}$ \\
1 & 0 & 1 & 0 & 1 & $-1.8 \mathrm{~V}$ & $\mathrm{R}_{\mathrm{L}}$ to $\mathrm{R}_{\mathrm{H}}$ \\
\hline
\end{tabular}

* ' 0 '- logic low; ' 1 '- logic high, and ' $\mathrm{X}$ ' - unknown or don't care.

TABLE II. SIZING OF INV $\mathrm{I}_{1} \mathrm{AND}_{1}$

\begin{tabular}{c|c|c}
\hline P/N Ratio & PMOS/NMOS in $I N V_{1}$ & $\boldsymbol{Q}_{\mathbf{1}}$ \\
\hline \hline \multirow{2}{*}{2} & $720 \mathrm{~nm} / 360 \mathrm{~nm}$ & $18 \times 220 \mathrm{~nm}$ \\
& $440 \mathrm{~nm} / 220 \mathrm{~nm}$ & $16 \times 220 \mathrm{~nm}$ \\
\hline \multirow{2}{*}{1} & $360 \mathrm{~nm} / 360 \mathrm{~nm}$ & $12 \times 220 \mathrm{~nm}$ \\
& $220 \mathrm{~nm} / 220 \mathrm{~nm}$ & $11 \times 220 \mathrm{~nm}$ \\
\hline \multirow{2}{*}{0.5} & $360 \mathrm{~nm} / 360 \mathrm{~nm}$ & $9 \times 220 \mathrm{~nm}$ \\
& $220 \mathbf{n m} / 440 \mathbf{n m}$ & $9 \times 220 \mathbf{n m}$ \\
\hline
\end{tabular}

signals. The write driver uses these signals to control two pairs of NMOS and PMOS switches and supply training voltage pair $V_{\text {top }}$ and $V_{\text {bot }}$. The training pair is applied to the two terminals of the memristor in the synapse design.

Determined by the training enable signal $E$, the training circuit can work under two modes.

- Operating mode: When $E=0$, the synapse is under regular operating (read) mode, and the training circuit is disabled.

- Training mode: The training circuit is enabled when $E=1$. By comparing the current synapse output $V_{\text {out }}$ and the expected $D_{\text {train, }}$, the training circuit generates $V_{\text {top }}$ and $V_{\text {bot }}$ applied to the two terminals of memristor to update or keep its memristance. We define $V_{\text {mem }}=V_{\text {top }}-V_{\text {bot }}$.

The training operation conditions are summarized in TABLE I.

Figure 3(b) depicts the proposed memristor-based synapse integrated with training circuit. An extra NMOS transistor $Q_{2}$ is inserted in synapse to isolate training operation from other voltage sources: when $E=1, Q_{2}$ is turned off so that the two terminals of memristor are controlled only by the training circuit, not affected by $V_{\text {in }}$.

The timing diagram of training circuit is demonstrated Figure 4(a). Before a training procedure starts, a sensing step is required to detect the current $V_{\text {out }}$ to be compared with $D_{\text {train }}$. In the sensing phase, accordingly, training enable signal $E$ is set to low for a very short period of time (e.g., 4.5ns) at the beginning of training. At the same time, $\overline{V_{\text {out }}}$ is sent to Latch, whose output $\overline{V_{\text {out }}}$, remains constant during one training period, as shown in Figure 3(a). In the training phase, $E$ is set back to high for a much longer time (i.e., $51 \mathrm{~ms}$ ) to change the memristance if needed.
We tested the training procedure by using the $\mathrm{TiO}_{2}$ memristor model [5]. The training circuit was designed by using TSMC $0.18 \mu \mathrm{m}$ technology with $V_{\mathrm{DD}}=1.8 \mathrm{~V}$. Changing memristance from $R_{H}$ to $R_{L}$ or verse vice takes about $51 \mathrm{~ms}$. The simulation result is shown in Figure 4(b). Here, the memristance is initialized as $\mathrm{M}=16 \mathrm{~K} \Omega$. In the first $51 \mathrm{~ms}$, it is trained to $1 \mathrm{~K} \Omega$ by setting $D_{\text {train }}$ to low. Then at $t=51 \mathrm{~ms}$, we set $D_{\text {train }}$ to high and train the memristance back to $R_{H}$ in the following $51 \mathrm{~ms}$.

\section{2) Asymmetry Gate Design}

As we mentioned in section III, the size of $Q_{I}$ affects the range of $V_{\text {out }}$. Instead of adding buffer or having giant $Q_{l}$ in synapse, the asymmetry gate design can be adopted to minimize the layout area of synapse design. More specifically, we tuned $\mathrm{P} / \mathrm{N}$ ratio of $I N V_{l}$ in the training circuit (see Figure 3(a)). TABLE II summarizes the required sizes of $I N V_{1}$ and $Q_{1}$ under different combinations that can make training successful. The result shows that the asymmetric design with $\mathrm{P} / \mathrm{N}$ ratio $=0.5$ can obtain the smallest area. The last option is used in the following synapse analysis.

\section{3) Multi-synapse Training Scheme}

Most of the neuron systems are constructed by multiple synapses. In this section, we discuss the corresponding training scheme by taking a 2-synapse neuron in Figure 5 as the example. Here, $A_{1}$ and $A_{2}$ are two synapse inputs received from other neurons. $M_{1}$ and $M_{2}$ are memristor-based weights for two synapses $S_{1}$ and $S_{2} . N$ is denoted for neuron with output $V_{\text {out }}$. The value of $V_{\text {out }}$ depends on the functionality of $N$

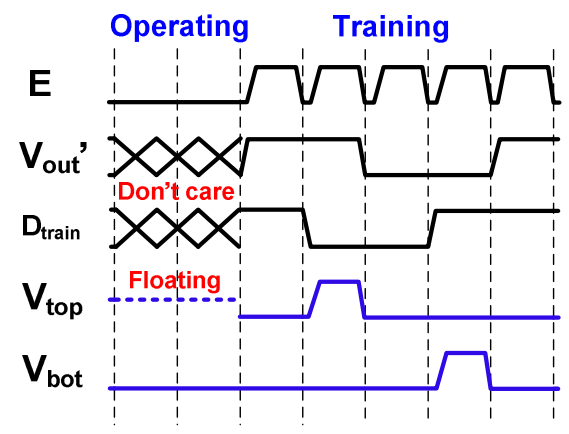

(a)

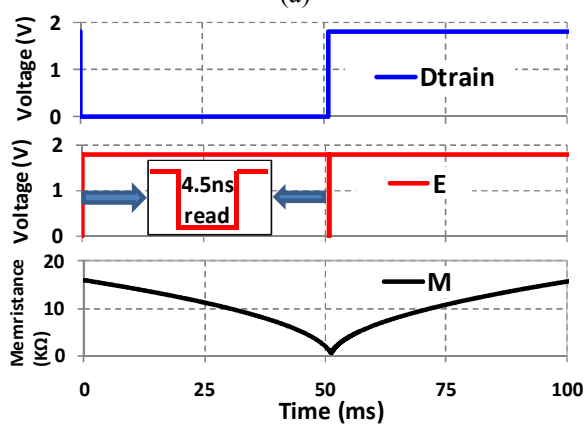

(b)

Figure 4: (a) The timing diagram of training circuit. (b) The simulation result of memristor training. 


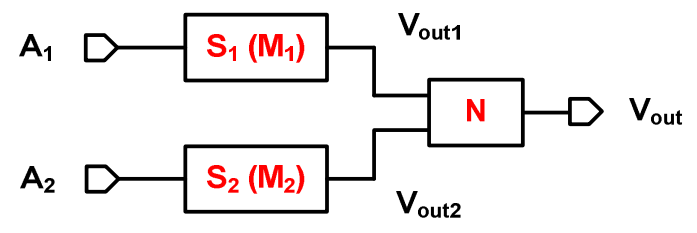

Figure 5: Two-input neuron structure.

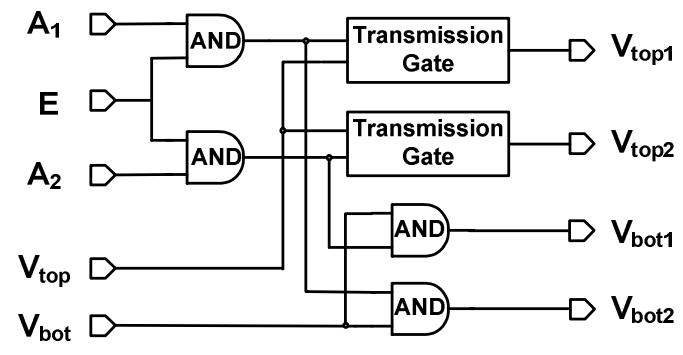

Figure 6:Training sharing distribution circuit.

as well as $V_{\text {out } 1}$ and $V_{\text {out } 2}$ from the two synapses. With the different combinations of $M_{1}$ and $M_{2}$, the two-input neuron could obtain different functionality.

To save design cost, memristances of the 2-synapse can be trained separately and share one training circuit. Figure 6 shows a training sharing distribution circuit, which generates training signals to control $M_{1}$ and $M_{2}$. The training sharing circuit operations under different conditions are shown in TABLE III.

The two synapse inputs $A_{l}$ and $A_{2}$ can be used to determine which memristor, $M_{1}$ or $M_{2}$, is in training. TABLE IV lists the required $A_{1}$ and $A_{2}$, when the logic functionality of $N$ is one of the following: OR/NOR, XOR/XNOR, AND/NAND.

Compared to the separated training circuit for each memristor, the shared scheme can reduce $26 \%$ of training circuit transistor count. More saving in cost and area can be obtained when utilizing this training sharing distribution scheme to multi-synapse structure with more inputs.

\section{4) Self-Training Mode}

To improve training time and reduce power consumption, we introduce the concept of self-training in our design: rather than using a fixed long training period (i.e., $51 \mathrm{~ms}$ ), the self-

TABLE III. TRAINING SHARING CIRCUIT OPERATION

\begin{tabular}{c|c|c|c|c}
\hline Status & $\boldsymbol{V}_{\text {top } 1}$ & $\boldsymbol{V}_{\text {bot } 1}$ & $\boldsymbol{V}_{\text {top } 2}$ & $\boldsymbol{V}_{\text {bot } 2}$ \\
\hline \hline Training $M_{I}$ & $V_{\text {top }}$ & $V_{b o t}$ & Floating & 0 \\
Training $M_{2}$ & Floating & 0 & $V_{\text {top }}$ & $V_{b o t}$ \\
\hline
\end{tabular}

TABLE IV. SYNAPSE INPUT PAIRS FOR DIFFERENT LOGICS

\begin{tabular}{c|c|c}
\hline Functionality of $\boldsymbol{N}$ & Training $\boldsymbol{M}_{\boldsymbol{1}}$ & Training $\boldsymbol{M}_{\mathbf{2}}$ \\
\hline \hline OR/NOR & $A_{l}=1, A_{2}=0$ & $A_{1}=0, A_{2}=1$ \\
XOR/XNOR & $A_{1}=1, A_{2}=0$ & $A_{1}=0, A_{2}=1$ \\
AND/NAND & $A_{1}=1, A_{2}=1$ & $A_{l}=1, A_{2}=1$ \\
\hline
\end{tabular}

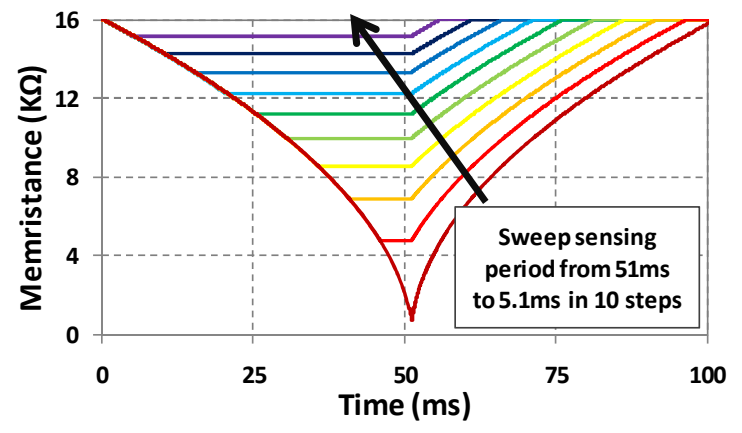

Figure 7: Self-training simulation.

TABLE V. SynAPSE POWER CONSUMPTION ANALysis

\begin{tabular}{c|c|c|c}
\hline \multicolumn{2}{c|}{ Operation } & Power & Energy \\
\hline \hline \multirow{2}{*}{ Read } & $R_{L}$ & $1.04 \mathrm{~mW}$ & $4.68 \mathrm{pJ}$ \\
& $R_{H}$ & $113.4 \mathrm{uW}$ & $0.51 \mathrm{pJ}$ \\
\hline \multirow{2}{*}{ Training } & From $R_{H}$ to $R_{L}$ & $216.7 \mathrm{uW}$ & $11.1 \mathrm{uJ}$ \\
& From $R_{L}$ to $R_{H}$ & $234 \mathrm{uW}$ & $11.9 \mathrm{uJ}$ \\
\hline
\end{tabular}

training mode automatically stop programming memristor whenever $V_{\text {out }}$ and $D_{\text {train }}$ become same.

The proposed training circuit supports self-training mode by dividing a long training period into multiple shorter periods and detecting $V_{\text {out }}$ in between. The programming period needs to be carefully selected: if it is too short, the delay and energy overheads induced by $V_{\text {out }}$ detection may overwhelm the benefit of self-training. On the contrary, a long programming period cannot show enough benefit.

The simulation result in Figure 7 shows the memristance changing when sweeping programming period from $5.1 \mathrm{~ms}$ to $51 \mathrm{~ms}$ in 10 steps. Obviously, the self-training mode could significantly reduce training time. In theory, the proposed training circuit can train the memristance to any value between $R_{H}$ and $R_{L}$. The real training time is determined by the specific application and neuron functionality.

\section{5) Power Analysis}

Reading and training simulation are conducted and power consumption data is collected in Table V. Energy is obtained when setting read time and write time as $4.5 \mathrm{~ns}$ and $51 \mathrm{~ms}$, respectively.

\section{CONCLUSION}

Memristor has been proven as a promising device in neuromorphic architecture for its high-density, nonvolatility, and unique memristive characteristic. In this paper, we proposed a memristor-based synapse that can be used in neuromorphic computing architecture. The corresponding training operations including multi-synapse schemes and selftraining have also been explored and discussed. The proposed synapse design can be generalized to other memristor materials for more applications. Next, we plan to further 
explore and utilize the analog properties of the proposed synapse and develop the memristor synapse based neuron network.

\section{ACKNOWLEDGMENTS AND DISCLAIMER}

Contractor acknowledges Government support in the publication of this paper. This material is based upon work funded by AFRL under contract No. FA8750-11-2-0046. Any opinions, findings and conclusions or recommendations expressed in this material are those of the author(s) and do not necessarily reflect the views of AFRL.

\section{REFERENCES}

[1] J. Partzsch, R. Schuffny, "Analyzing the Scaling of Connectivity in Neuromorphic Hardware and in Models of Neural Networks," IEEE Transactions on Neuron Networks, pp. 929-935, 2011.

[2] M. Wang, B. Yan, J. Hu, P. Li, "Simulation of large neuronal networks with biophysically accurate models on graphics processors," International Conference on Neural Networks, pp. 3184-3193, 2011.

[3] H. Shayani, P.J. Bentley, A.M.Tyrrell, "Hardware Implementation of a Bio-plausible Neuron Model for Evolution and Growth of Spiking Neural Networks on FPGA," NASA/ESA Conference on Adeptive Hardware and Systems, pp. 236-243, 22-25 Jun. 2008.

[4] L. Chua, "Memristor-the missing circuit element," IEEE Trans. on Circuit Theory, vol. 18, pp. 507-519, 1971.
[5] D. B. Strukov, et al., "The missing memristor found," Nature, vol. 453, pp. 80-83, 2008.

[6] X. Wang, et al., "Spintronic memristor through spin-torqueinduced magnetization motion," IEEE Electron Device Letters, vol. 30, pp. 294-297, 2009.

[7] S. H. Jo, T. Chang, I. Ebong, B. B. Bhadviya, P. Mazumder, and W. Lu, "Nanoscale Memristor Device as Synapse in Neuromorphic Systems,"Nano Letters, vol. 10, no. 4, pp. 1297 1301, March 2010.

[8] Y. Ho, G. Huang, P. Li, "Nonvolatile memristor memory: Device characteristics and design implications," IEEE/ACM International Conference on Computer-Aided Design (ICCAD), pp. 485-490, 2009.

[9] J. Cong, B. Xiao, "mrFPGA: A novel FPGA architecture with memristor-based reconfiguration," IEEE/ACM Internaltional Symposium on Nanoscale Architectures (NANOARCH), pp. 18,2011

[10] D. B. Strukov, et al., "Hybrid CMOS/Memristor Circuits," IEEE International Symposium on Circuits and Systems (ISCAS), pp. 1967-1970, 2010.

[11] J. Rajendran, H. Manem, R. Karri, and G. Rose, "Memristor based programmable threshold logic array," IEEE/ACM International Symposium on Nanoscale Architecture, pp. 5-10, Jun. 2010.

[12] G. Rose, R. Pino, Q. Wu, "Exploiting Memristance for LowEnergy Neuromorphic Computing Hardware," IEEE International Symposium on Circuits and Systems (ISCAS), pp. 2942-2945, 2011. 